\title{
A DIELECTRIC LOADED SLOW WAVE STRUCTURE FOR SEPARATION OF RELATIVISTIC PARTICLES'*
}

C. T. M. Chang, J. W. Dawson, and R. L. Kustom

Argonne National Laboratory

Argonne, Illinois

Summary

Dielectric loaded waveguides of rectangular cross section are considered as synchronous traveling wave particle separators. These structures support two linearly independent modes: those which have no electric field perpendicular to the dielectric, and those which have no magnet:c field perpendicular to the dielectric. Both modes have suitable transverse deflection forces with phase velocities which can be slower than the speed of light.

Degeneracies and dispersion relations are discussed. The theoretical conclusions are supported by experimental studies which include slotted line and bead perturbation mea surements.

\section{Synchronous Traveling Wave Separator}

In a synchronous particle separator, as decribed by Montague, 1 the phase velocity of the electromagnetic wave is adjusted to be identical with the velocity of the wanted particle. Viewed in a frame of reference which is moving at this velocity, the wanted particle appears stationary at the RF phase angle it encountered at the time of entry into the structure. The wanted particle undergoes continuous transverse deflection while in the structure. The unwanted particles have a velocity which is different from the wanted particles and consequently tend to slip forward or backward in RF phase; Fig. 1 illustrates this action. If the length of the structure is chosen to allow the unwanted particles to go through at least $2 \pi$ radians of RF phase, the unwanted particles leave the structure with little or no deflection relative to a wanted particle which enters at a favorable phasc angle. The unwanted particles and those wanted particles which enter at an unfavorable phase angle are stopped in a stopping block downstream of the separator. The kinematics of such a device have been described in detail by Val'dner and Glazkov. 2

\section{Traveling Wave Structure Propagating Modes}

A dielectric loaded waveguide with rectangular cross section ${ }^{3}$ appears to meet the requirements for a traveling wave particle separator. There

* Work performed under the auspices of the U. S. Atomic Energy Commission. are two linearly independent modes which the structure can support. Both of these have transverse deflecting forces. The cross section of the waveguide and the coordinate system are shown in Fig. 2. A conducting plane can be placed at $y=0$, in which case there would be only one-half the waveguide. Although the latter structure is the one which was studied, the results apply equally well to the full structure with an even mode suppressor.

The modes $4,5,6$ which can be supported by the structure have either no electric field perpendicular to the dielectric, called the longitudinalsection electric (LSE), or no magnetic field perpendicular to the dielectric, called the longitudinal-section magnetic (LSM).

The components of the LSE modes are given by

$$
\overline{\mathrm{E}} \quad=-j \omega \mu,{ }_{0}\left\{\nabla \times \pi_{h} \hat{\mathrm{y}}\right\} \text {, }
$$

where $\pi_{h}$ is the Hertzian potential

$$
\pi_{h}=A_{m n} \cos \frac{m \pi x}{s} f_{n}(y) e^{-j h m n^{2},},
$$

and

$$
f_{n}(y)=\left\{\begin{array}{c}
\sin a_{n} \tau \sinh \gamma_{n} y \\
|y|<\ell \cdots \tau \\
\frac{y}{|y|} \sinh \gamma_{n}(\ell-\tau) \sin a_{n}(\ell-|y|) \\
\ell-\tau<|y|<\ell
\end{array}\right.
$$

The transverse deflection force is proportional to the $\mathrm{H}_{x}$ ficld component of this mode.

The field components of the LSM modes are given by

$$
\bar{H} \quad=j \omega \epsilon_{0} K(y)\left\{\nabla \times \pi_{e} \hat{y}\right\} .
$$

$$
\mathrm{W}(\mathrm{y})=\left\{\begin{array}{ll}
1 & |\mathrm{y}|<\ell-\tau \\
{ }_{\mathrm{r}} & \ell-\tau<|\mathrm{y}|<\ell,
\end{array},\right.
$$

and $\pi_{\mathrm{e}}$ is

$$
\text { "e }=B_{m n} \sin \frac{m \pi x}{s} g_{n}(y) e^{-j h} m n^{z} \text {, }
$$


and

$$
g_{n}(y)=\left\{\begin{array}{c}
\cos a_{n} \tau \cosh \gamma_{n} y \\
|y|<\ell-\tau \\
\frac{1}{\epsilon_{r}} \cosh \gamma_{n}(\ell-\tau) \cos a_{n}(\ell-|y|) \\
\ell-\tau<|y|<\ell .
\end{array}\right.
$$

In the LSM mode the deflectionforce in the transverse direction is proportional to $\left(E_{y}-v_{x}\right)$.

The distribution of force across the aperture in either the LSM or LSE rode for $m=1$ is given by

$$
F_{y}=F_{m} \sin \frac{\pi x}{s} \cosh y y .
$$

Dispersion Curves and Cutoff Frequencies

The modes which propagate must simultaneously satisfy the boundary conditions and the separation equations. With some rearrangement the separation equations yield

$$
a_{n}^{2}+\epsilon_{r} r_{n}^{2}=\left(\epsilon_{r}-1\right)\left[\left(\frac{m \pi}{s}\right)^{2}+h_{m n}^{2}\right] \text {, }
$$

and

$$
a_{n}^{2}+v_{n}^{2}=\left(e_{r}-1\right) k_{0}^{2}
$$

The bounda ry conditions at the dielectric interface give rise to a transcendental equation which, when solved simultaneously with Eqs. (1) and (2), gives the phasevelocity - frequency characteristic for the waveguide. The transcendental equation for the LSE mode is

$$
a_{n} \cot a_{n} \tau=-\gamma_{n} \operatorname{coth} \gamma_{n}(\ell-\tau),
$$

and for the LSM mode it is

$$
a_{n} \tan a_{n} \tau=E_{r} \gamma_{n} \tanh \gamma_{n}(\ell-\tau) .
$$

The value of $\gamma_{\mathrm{g}}$ can be imaginary, in which case the waveguide supports a fast wave mode.

The cutoff frequency of the various modes is found by setting $\mathrm{h}$ mn equal to zero in Eqs. (1) through (4). Figure 3 is a plot of Eqs. (1), (3), and (4) for an experimental section of waveguide. Any intersection between the curves for $\mathrm{Eq}$. (1) and Eqs. (3) and (4) corresponds to a cutoff frequency of a propagating mode.

Table $I$ is a compilation of the lowest frequency

\begin{tabular}{|c|c|c|}
\hline$f_{c}(\mathrm{GHz})$ & $\mathrm{LSM} / \mathrm{LSE}$ & $\mathrm{m}$ \\
\hline 1.7975 & LSM & 1 \\
\hline 3.4212 & LSM & 2 \\
\hline 3.6358 & LSE & 0 \\
\hline 3.9972 & LSE & 1 \\
\hline 4.0222 & LSM & 1 \\
\hline 4.7789 & LSM & 3 \\
\hline 4.8893 & LSE & 2 \\
\hline
\end{tabular}
modes for a guide with $\ell=3.4 \mathrm{~cm}, s=7.21 \mathrm{~cm}$, $\tau=1.25 \mathrm{~cm}$, and $\epsilon_{\mathrm{r}}=2.55$.
TABLE I

Cutoff Characteristics of a Dielectric Loaded Waveguide

\section{Power Requirements}

The power which flows down the waveguide is related to the deflecting fields through the integral of the Poynting vector,

$$
\mathrm{P}_{\mathrm{f}}=\frac{1}{2} \int_{0}^{S} \int_{-\ell}^{\ell}\left(\overline{\mathrm{E}} \times \overline{\mathrm{H}}^{*}\right) \cdot \hat{\mathrm{z}} \mathrm{dy} \mathrm{dx}
$$

Obviously, the power which propagates in the dielectric does not contribute to deflection and so represents an inefficiency in the structure. How ever, for L-and S-band structures which use dielectrics with a relativcly high dielectric con$\operatorname{stant}\left(\epsilon_{r}>3.0\right)$, this bypass power is of tolerable proportion.

Losses in the structure must be kept sufficiently small so that the unwanted particles do not receive a sizable deflection due to asymmetrical force interactions in passing through the RF cycle. The conductive losses in the waveguide walls are given by

$$
\mathrm{P}_{\mathrm{c}}=\frac{1}{2} \mathrm{R}_{\mathrm{s}} \iint_{\text {conducting walls }} \hat{\mathrm{n}} \times\left.\overline{\mathrm{H}}\right|^{2} \mathrm{ds},
$$

where $R_{S}$ is the surfacc resistance of conducting walls and $\hat{n}$ is the normal vector to walls. The dielectric losses are given by

$$
\mathrm{P}_{\mathrm{d}}=\frac{1}{2} \omega \epsilon_{\boldsymbol{r}} \mathrm{E}^{\prime \prime} \iiint_{\mathrm{Vol} .} \overline{\mathrm{E}} \cdot \overline{\mathrm{E}}^{-k} \mathrm{dv} .
$$

From the lattcr it is obvious that a low luss dielectric is necessary. Either aluminum oxide or bervllium oxide meets the necessary require ments of low loss and high dielectric constant.

\section{Aperture}

Theaperture can belimited in two ways. From Eq. (5) it can be seen that the required power is directly related to the size of the aperture. A typical high power klystroncan deliver up to about 
$10 \mathrm{MW}$ for 40 to $50 \mu \mathrm{s}$ duration. Thus for a given field, i.e., deflection, the aperture is limited by the available power.

The second aperture limit is created by the onset of degerieracies. Increasing the cross section of the waveguide without changing the frequency tends to lower the cutoff frequencies of the higher order modes. These will divert power from the primary mode and $m$ ight have adverse interaction with the particle beam.

\section{Experimental Studies}

The experimental arrangement used to dete $\mathbf{r}$ mine the phase velocity - frequency characteristic is shown in Fig. 4. The distance between nulls in the standing wave pattern corresponds to one-half the waveguide wavelength. The phase velocity is the product of the frequency and twice the distance between nulls. The calculated and measured phase velocity - frequency curves for an LSM $_{10}$ mode are shown in Fig. 5.

A modal expansion for this system indicates that no LSE modes or even LSM modes will be excited by a centrally located y-directed cur rent probe. From Table $I$ it is apparent that no other modes should be excited before the LSM $_{11}$ mode at about $4 \mathrm{GHz}$. This action is verified by the slotted line measurements. Figure 6a shows the standing wave pattern below the cutoff frequency of the $\mathrm{LSM}_{11}$ mode, and Fig. 6b shows the pattern above the $\mathrm{LSM}_{11}$ cutoff. The $\mathrm{LSM}_{10}$ and $\mathrm{LSM}_{11}$ modes in Fig. $6 \mathrm{~b}$ are verified by wavelength measurements.

Field patterns were verified by perturbation measurements in a cavity which had the same cross section as the waveguide and was made $4.38 \mathrm{~cm}$ long. The resonant frequency was 3.296 GHz, which is in good agreenent with the LSM $_{10}$ waveguide calculations. Figure 7 shows the shift in resonant frequency as a function of $y$-position of the bead. The $x$ - and $z$-positions were centered in the guide. A calculated curve is also plotted from the following formula:

$$
f=f_{1}+\left[\frac{\cosh ^{2} \gamma y-\cosh ^{2} \gamma y_{1}}{\cosh ^{2} \gamma y_{2}-\cosh ^{2} \gamma y_{1}}\right]\left(f_{2}-f_{1}\right) \text {. }
$$

The values of $f_{2}$ and $f_{1}$ are chosen to normalize the predicted curve to the data.

\section{Trajectory Studies}

A computer program for the $360 / 75$ was written which calculates particle trajectories through the separator, including cross coupling between $x$ - and $y$-forces. The program divides the separator length into increments and calculates the motion due to the field on an incremental basis.

As a typical separator example, a 6.5 in. $x$ 3. $94 \mathrm{in.}$ waveguide operating in the $\mathrm{LSM}_{10}$ mode at $1.42 \mathrm{GHz}$ was chosen. The effective aperture for particle separation is $3 \mathrm{in.} \times 3 \mathrm{in.} \mathrm{A} 1.41$ $\mathrm{GeV} / \mathrm{c}$ kaon can receive a deflection greater than $1.0 \mathrm{mrad}$ in a $4.0 \mathrm{~m}$ structure with $7.5 \mathrm{MW}$ of synchronous propagating power. The calculated attenuation constant is 0.004 per meter.

The deflection results are summarized in the phase plane diagrams shown in Fig. 8. At entry $\mathrm{K}^{-}, \overline{\mathrm{p}}$, and $\pi^{-}$have the same distribution. At the exit of the separator the limiting phase plane diagrams for kaons are shown in Fig. 8a. The diagrams for pions and antiprotons are shown in Fig. $8 \mathrm{~b}$. The aberrations due to variation of deflecting force with $\mathrm{x}$-positions are included in the last figure.

\section{Discussion}

In a separated beam, either the LSM or LSE mode could be used. IIowever, the LSM 10 mode is usually the dominant mode and for reasonable apertures would operate at L-band. For momenta greater than $1.5 \mathrm{GeV} / \mathrm{c}$, the required structure length tends to be too large and so the LSE mode becomes more appealing. However, operation in this mode does require mode filtering to prevent degeneracies.

Several types of mode filters without loss of propagating bandwidth would appear possible. Experiments are being performed which will test their success.

A reasonable bandwidth is desirable so that the separator can be used over a wide momentum range. This is accomplished by operating at different frequencies with a tunable klystron. The dispersion curve of the dielectric loaded structure is ideally suited to this type of operation. Particle velocities between $0.9 \mathrm{c}$ and $1.0 \mathrm{c}$ can be matched by the phase velocity of the wave with a $\pm 10 \%$ frequency variation.

\section{Conclusions}

We feel that a synchronous particle separator using a dielectric loaded rectangular structure is practical and, for low momentum beams, offers several advantages over electrostatic separators. While it would appear that available klystron power would limit the use of these separators to bubble chamber beams, our analysis indicates 
that a synchronous separator excited as a cavity would be usable for some counter experiments. Our interests at the moment involve the use of a synchronous separator in a very short 0.5 to 1.5 GeV/c kaon beam.

\section{Acknowledgments}

We acknowledge the many sage comments and suggestions by Alfred Moretti of Argonne National Laboratory and the efforts of Duane Amundson, who built the hardware and performed the perturbation and interference measurements.

\section{References}

1. B. W. Montague, CERN-PS/Int. AR/PSep/ $60-1(1960)$.
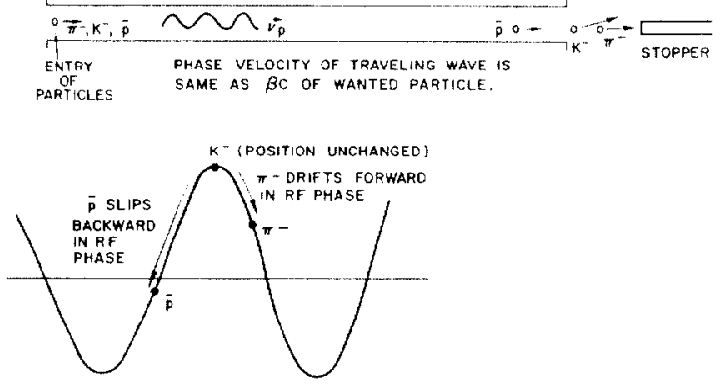

PARTICLE VELOCITY IN MOVING FRAME OF REFERENGE WITH WANTED PARTICLE YELOCITY.

Fig. 1 - Particle separation in traveling wave separator

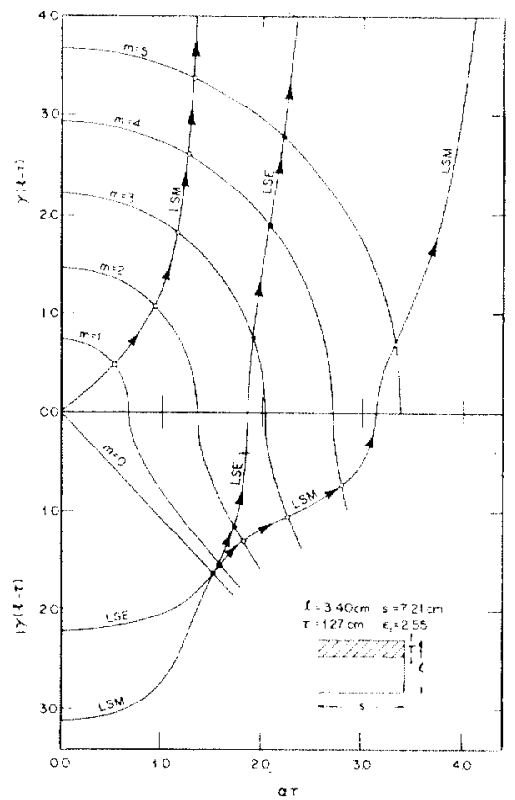

Fig. 3 - Plot of separation equation at cutoff ( $\left.h_{m n}=0\right)$ for various $m$ and the LSM and LSE transcendental equations for boundary interface
2. O. A. Val'dner and A. A. Glazkov, Instr. and Expr. Tech. 4. 1326 (1965)

3. J. W. Dawson and R. L. Kustom, "A Single Section Traveling Wave Particle Separator, " Argonne National Laboratory internal report JWD/RLK-1 (June 17, 1968).

4. R. F. Harrington, Time Ha rmonic Elcctromagnetic Fields, 158-171, McGraw-Hill, New York (1961).

5. L. Pincherle, Phys. Rev. 66, 5 and 6, 118 (1944).

6. R. L. Collin, Field Theory of Guided Waves chapter 6, McGraw-Hill, New York (1960).

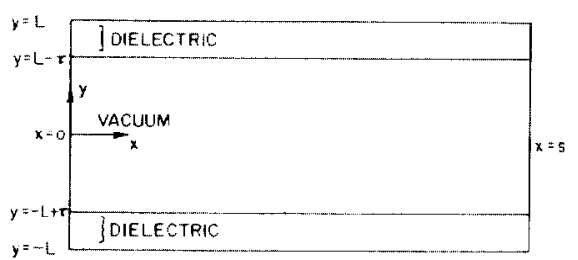

Fig, 2 - Geometry and coordinate system of dielectric loaded waveguide

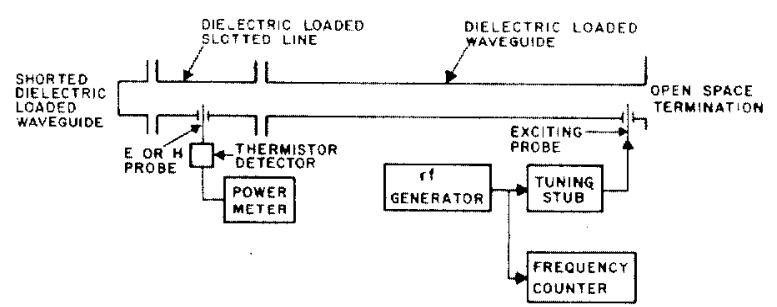

Fig. 4 - Experimental apparatus for determining phase velocities

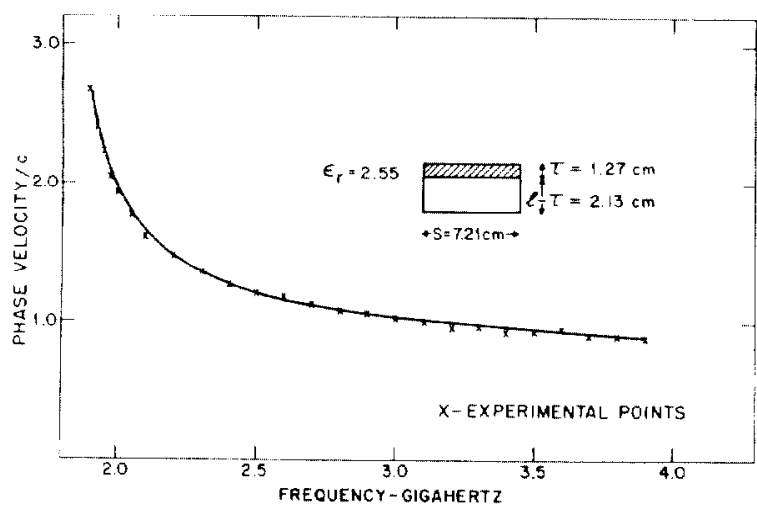

Fig. 5 - Phase velocity - frequency curves for an $\operatorname{LSM}_{10}$ mode 


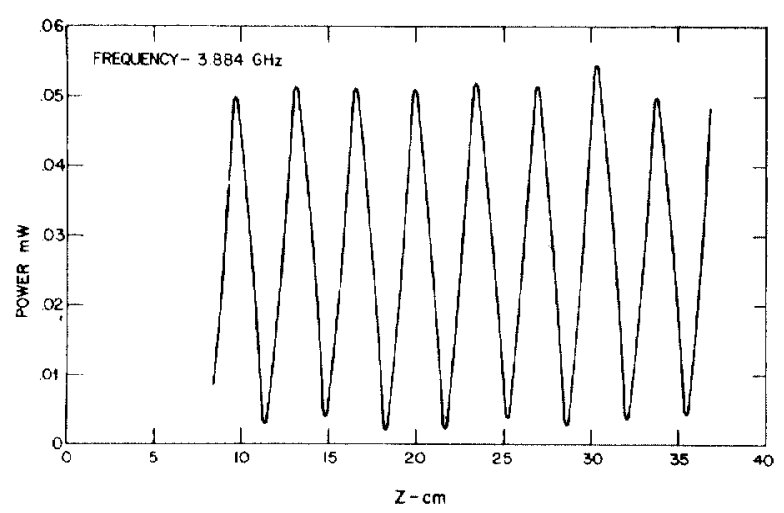

Fig. ba - Standing wave pattern below cutoff of LSM $_{11}$ mode

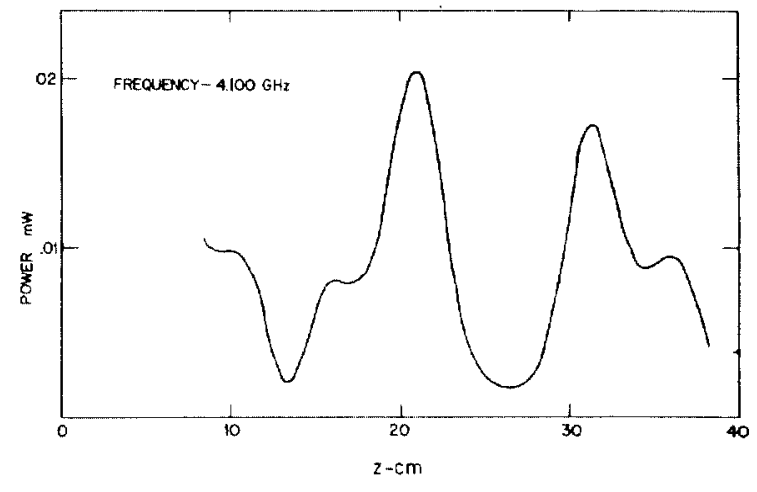

Fig. 6b - Standing wave pattern above cutoff of LSM $_{11}$ mode

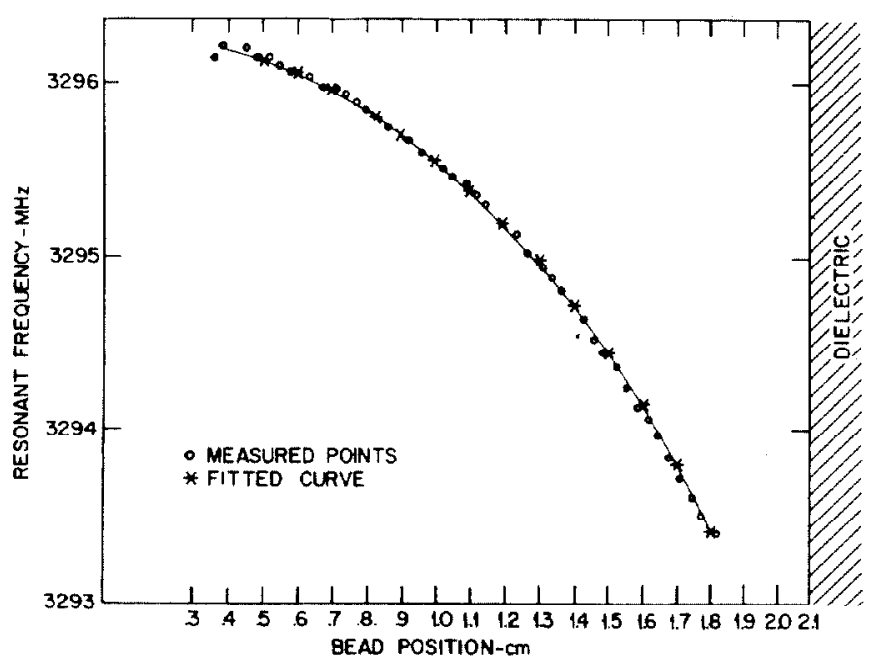

Fig. 7 - Shift in resonant frequency of a dielectric

loaded cavity as a function of bead position

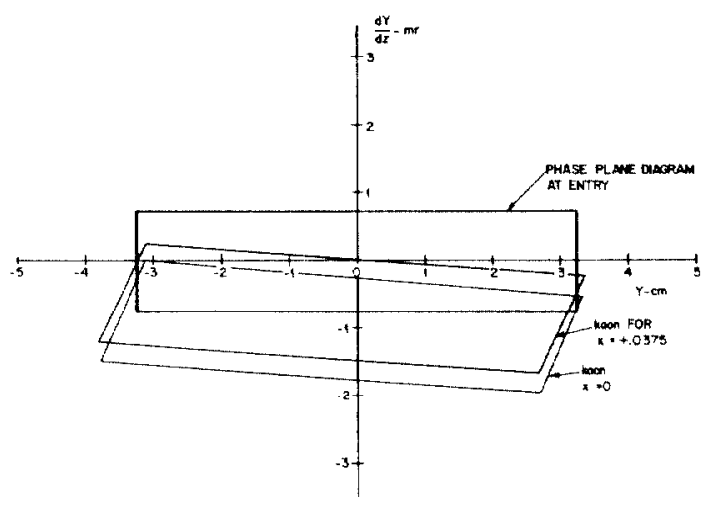

Fig, 8a - Phase plane diagram at entrance to and exit from traveling wave separator for kaons

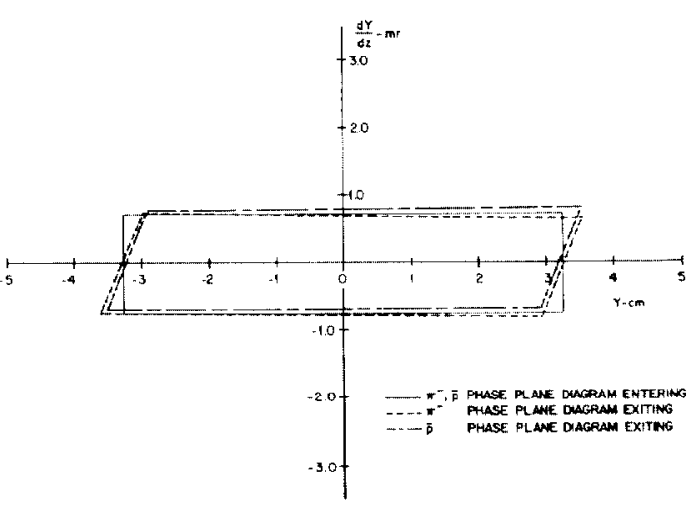

Fig. 8b - Phase plane diagram at entrance to and exit from traveling wave separator for pions and antiprotons 